\title{
Large eddy simulations of spatially growing subsonic and supersonic turbulent round jets
}

\author{
MOHAMED MAIDI and MARCEL LESIEUR* \\ Equipe MoST/LEGI, B.P. 53, 38041 Grenoble Cedex 09, France
}

\begin{abstract}
This paper presents an application of large eddy simulations (LESs) using the filtered structure function model to spatially developing compressible round jets issuing from a perturbed upstream velocity profile close to a top hat. For centreline Mach number $M=0.9$ and Reynolds number $R e=3600$, the numerical solution compares satisfactorily against a forced-jet direct numerical simulation (DNS) and experimental data, both previously reported. High Reynolds number ( $R e=36000)$ 'free' jets at Mach 0.7 (case 1) and 1.4 (case 2) are studied. Here, an isotropic random white-noise perturbation is superposed on the upstream velocity. The Mach 0.7 jet has a convective Mach number of 0.35 , and is weakly affected by compressibility. In this case, axisymmetric vortex rings are first shed from the nozzle and undergo alternate pairing further downstream. Then turbulence develops. The centreline velocity decay and some other statistical quantities are, in the self-similarity region, in very good agreement with previous incompressible experiments. At Mach 1.4, an impressive upstream reduction of the jet spreading rate is observed, due to an important delay of Kelvin-Helmholtz instability due to compressibility effects. Alternate pairing occurs immediately, and vortices are much more elongated in the flow direction. Further downstream, the jet becomes subsonic, develops into turbulence and spreads out again at a rate comparable with its subsonic counterpart. The potential-core length is increased by $27 \%$ from the subsonic to the supersonic case. This is in agreement with several laboratory experiments. Finally, the effects of Mach number increase upon various statistical quantities such as Reynolds stresses and radial lengthscale are studied. Results compare favourably against some experiments and temporal DNSs. From the point of view of Lumley's anisotropy invariant map evaluated on the whole physical domain, the Mach 0.7 jet is dominated by axisymmetric structures and the Mach 1.4 jet by streamwise perturbations.
\end{abstract}

\section{Introduction}

Due to their wide range of industrial applications in propulsion, combustion and acoustics, round jets have given rise to numerous analytical, experimental and numerical studies. Extensive research has been carried out in order to provide a more complete understanding of jet dynamics and a detailed picture of flow structures governing the spatial evolution. Experiments concerning incompressible round jets have demonstrated that for a broad range of Reynolds numbers, jet dynamics is dominated by large-scale toroidal vortices in the transitional regime downstream of the nozzle [1, 2]. These vortices result from the development of Kelvin-Helmholtz (KH) instability (axisymmetric mode) and evolve further downstream under the effect of other instability modes such as the helical mode whose amplification rate may be larger than that of the axisymmetric mode. The formation and downstream evolution of these vortices play a significant role in the jet's spatial development and spreading rate. It is therefore of interest to understand their dynamics, as well as their role in mixing

\footnotetext{
*Corresponding author. E-mail: Marcel.Lesieur@hmg.inpg.fr
} 
or aerodynamic noise generation. Since the nature and topology of these coherent vortices depend strongly upon the jet inlet conditions existing in the experiments, it is very important to investigate the effect of different inlet parameters.

In the present study we focus on the effects of compressibility effects on the spatial development of turbulent round jets in an ideal gas. This is achieved by varying (up to 1.4) the Mach number imposed at inlet conditions. In a plane mixing layer between two parallel flows of velocities $U_{1}$ and $U_{2}$, the theoretical results of Bogdanoff [3] identified the convective Mach number $M_{\mathrm{c}}$ as the relevant parameter characterising compressibility effects. When compressibility is not too high, it is equal to $\Delta U /\left(c_{1}+c_{2}\right)$, where $\Delta U=U_{1}-U_{2}$ and $c_{1}$ and $c_{2}$ are the sound speeds in each layer. Papamoschou and Roshko [4] showed experimentally the importance of $M_{\mathrm{c}}$ on the mixing layer growth. The effect of $M_{\mathrm{C}}$ on Reynolds stresses was considered in experiments camed out by Elliott and Samimy $[5,6]$. One can also define $M_{\mathrm{C}}$ for an annular mixing layer, and its influence on pressure fluctuations was considered in a temporal study of using DNS [7]. Several works [4, 8-11] have displayed evidence that the flow structures of planar mixing layers are dramatically affected by the increase of $M_{\mathrm{c}}$. These studies indicate that at low $M_{\mathrm{c}}\left(M_{\mathrm{c}} \leq 0.5\right)$, coherent vortices are similar to those observed in the incompressible case. The pairing of these vortices is the principal cause of shear-layer linear growth [12]. As $M_{\mathrm{c}}$ exceeds a value of the order of 0.5 , turbulence structures become more three-dimensional, of smaller scale and less coherent. The pairing process of the eddies is then difficult to identify. Linear-stability analysis and direct numerical simulations (DNSs) $[13,14]$ showed that the growth rate and turbulence levels were reduced by increasing convective Mach numbers. In these DNSs, the mixing layer was forced quasi two-dimensionally: pairing of straight $\mathrm{KH}$ vortices was observed at $M_{\mathrm{C}}=0.4$, whereas they transformed into large staggered lambda-shaped vortices superposing on each other at $M_{\mathrm{C}}=0.8$ and 1.05. Fouillet [5] performed a similar simulation, but with an initial random isotropic three-dimensional perturbation. At low $M_{\mathrm{c}}$ he found the helical-pairing vortex organization discovered in the incompressible case by Comte et al. [16], but recovered Sandham and Reynolds' patterns above $M_{\mathrm{c}}=0.7$. In this respect, it seems that helical pairing that occurs in a natural plane mixing layer is inhibitted above a convective Mach number of this order of magnitude.

Effects of compressibility upon round-jet structures have been experimentally studied [1719]. At low compressibility $\left(M_{\mathrm{c}}<0.5\right)$, these works demonstrated the emergence of ring-like axisymmetric vortices in the transitional region. At high compressibilty $\left(M_{\mathrm{c}}>0.6\right)$, the turbulent structures become increasingly three dimensional and less coherent. These experiments, as well as the DNSs carried out by Freund et al. [7]), found that the significant effect of compressibility on the annular mixing layer is a reduced growth rate. This is in agreement with planar mixing layer studies. Let us mention also the DNS of uniformly sheared flow done by Sarkar [20], who stresses that reduced turbulent production is responsible for a decrease in turbulence level. This result was also observed by Simone et al. [21] in their DNS of the same flow. In addition to the reduction in the turbulent fluctuations, Freund's numerical simulations and our present study show the inhibition of radial turbulent length scale with increasing Mach number.

Here, we will study subsonic and supersonic turbulent jets with the aid of large eddy simulation (LES) techniques. This allows one to reach high Reynolds numbers with a moderate number of grid points. LESs are hence of very low cost with respect to DNSs at the same Reynolds number. We will provide ample validations of our calculations against DNS and laboratory experiments. Concerning the upstream nozzle, it is just replaced by a jet-like inflow velocity profile to which various random perturbations are superposed. Notice that in the supersonic case, shocks and Mach waves cannot be produced since, contrary to a real-nozzle case, no obstacle is present in the flow. But the study is of interest as far as coherent-vortex dynamics are concerned. 


\section{Governing equations}

The governing equations for this flow are the compressible Navier-Stokes equations written in a Cartesian frame, in the so-called fast-conservation form

$$
\frac{\partial \mathbf{U}}{\partial t}+\frac{\partial \mathbf{F}_{\mathbf{i}}}{\partial x_{i}}=0
$$

In which $\mathbf{U}$ is a five-component vector defined as

$$
\mathbf{U}={ }^{\mathrm{T}}\left(\rho, \rho \mathbf{u}_{1}, \rho \mathbf{u}_{2}, \rho \mathbf{u}_{3}, \rho \mathbf{e}\right)
$$

Such a formulation (1) includes the continuity, momentum and total energy equations. The total energy $\rho e$ is defined as (neglecting gravity)

$$
\rho e=\rho C_{v} T+\frac{1}{2} \rho\left(u_{1}^{2}+u_{2}^{2}+u_{3}^{2}\right)
$$

For a Newtonian fluid, the fluxes $\mathbf{F}_{\mathbf{i}}$ are

$$
\mathbf{F}_{\mathbf{i}}=\left(\begin{array}{l}
\rho u_{i} \\
\rho u_{i} u_{1}+p \delta_{i 1}-2 \mu S_{i 1} \\
\rho u_{i} u_{2}+p \delta_{i 2}-2 \mu S_{i 2} \\
\rho u_{i} u_{3}+p \delta_{i 3}-2 \mu S_{i 3} \\
(\rho e+p) u_{i}-2 \mu u_{i} S_{i j}-k \partial T / \partial x_{i}
\end{array}\right)
$$

where $k$ is the thermal conductivity, $\mu$ the viscosity and $S_{i j}$ the deviatoric part of the deformation tensor:

$$
S_{i j}=\frac{1}{2}\left(\frac{\partial u_{i}}{\partial x_{j}}+\frac{\partial u_{j}}{\partial x_{i}}-\frac{2}{3}(\vec{\nabla} \cdot \vec{u}) \delta_{i j}\right)
$$

The viscosity is computed using Sutherland's law:

$$
\mu(T)=\mu\left(T_{0}\right)\left(\frac{T}{T_{0}}\right)^{0.5} \frac{1+S / T_{0}}{1+S / T}
$$

where $\mu\left(T_{0}\right), T_{0}$ and $S$ are gas dependents. The Prandtl number is assumed constant and equal to 0.72 .

\subsection{Large eddy simulation}

The LES equations are obtained by applying a low-pass spatial filter $G_{\Delta}$ of width $\Delta$ to the Navier-Stokes equations (1). This eliminates scales smaller than $\Delta$. Mathematically, the filtered field for any quantity $f$ is given by

$$
\bar{f}(\vec{x}, t)=\int f(\vec{y}, t) G_{\Delta}(\vec{x}-\vec{y}) \mathrm{d} \vec{y}
$$

Such a filter will be called a 'bar-filter'. Scales of motion are then decomposed into a gridscale $\bar{f}$ and subgrid-scale part $f^{\prime}$. The compressible LES formalism is simplified by the use of density-weighted Favre filtering (see [22] for details)

$$
\tilde{f}=\frac{\overline{\rho f}}{\bar{\rho}}
$$

We then have

$$
\overline{\mathbf{U}}={ }^{\mathrm{T}}\left(\bar{\rho}, \bar{\rho} \tilde{u}_{1}, \bar{\rho} \tilde{u}_{2}, \bar{\rho} \tilde{u}_{3}, \bar{\rho} \tilde{e}\right)
$$


and the bar-filtered total energy equation becomes

$$
\bar{\rho} \tilde{e}=\bar{\rho} C_{v} \tilde{T}+\frac{1}{2} \overline{\rho\left(u_{1}^{2}+u_{2}^{2}+u_{3}^{2}\right)}
$$

Bar-filtered fluxes $\overline{\mathbf{F}_{\mathbf{i}}}$ are:

$$
\overline{\mathbf{F}_{\mathbf{i}}}=\left(\begin{array}{l}
\overline{\rho \tilde{u}_{i}} \\
\overline{\rho u_{i} u_{1}}+\bar{p} \delta_{i 1}-2 \overline{\mu S_{i 1}} \\
\overline{\rho u_{i} u_{2}}+\bar{p} \delta_{i 2}-2 \overline{\mu S_{i 2}} \\
\overline{\rho u_{i} u_{3}}+\bar{p} \delta_{i 3}-2 \overline{\mu S_{i 3}} \\
\overline{(\rho e+p) u_{i}}-2 \overline{\mu u_{i} S_{i j}}-\overline{k \partial T / \partial x_{i}}
\end{array}\right)
$$

The bar-filtered state equation becomes (assuming $C_{p}, C_{v}$ and hence $R$ are constant)

$$
\bar{p}=\bar{\rho} R \tilde{T}
$$

From these expressions, it is natural to consider that the relevant variables for the compressible LES problem are $\bar{\rho}$ and the Favre-filtered quantities $\tilde{u}_{i}$ and $\tilde{e}$. Working with these variables, we define the subgrid stress tensor $\overline{\overline{\mathcal{T}}}$ as

$$
\mathcal{T}_{i j}=-\overline{\rho u_{i} u_{j}}+\bar{\rho} \tilde{u}_{i} \tilde{u}_{j}
$$

This tensor can be split into isotropic and deviatoric parts, the latter denoted $\tau_{i j}$ :

$$
\mathcal{T}_{i j}=\tau_{i j}+\frac{1}{3} \mathcal{T}_{l l} \delta_{i j}
$$

The bar-filtered total energy then reads

$$
\bar{\rho} \tilde{e}=\bar{\rho} C_{v} \tilde{T}+\frac{1}{2} \bar{\rho}\left(\tilde{u}_{1}^{2}+\tilde{u}_{2}^{2}+\tilde{u}_{3}^{2}\right)-\frac{1}{2} \mathcal{T}_{l l}
$$

Lesieur and Comte [22] introduced quantities called macro-pressure and macro-temperature, defined by

$$
\varpi=\bar{p}-\frac{1}{3} \mathcal{T}_{l l}
$$

and

$$
\vartheta=\tilde{T}-\frac{1}{2 C_{v} \bar{\rho}} \mathcal{T}_{l l}
$$

The bar-filtered equation of state is:

$$
\varpi=\bar{\rho} R \vartheta+\frac{3 \gamma-5}{6} \mathcal{T}_{l l}
$$

The ratio of the second to the first term of the r.h.s. of this equation may be shown to be of modulus proportional to $\gamma|3 \gamma-5| M_{\text {sgs }}^{2} / 6$, where $M_{\text {sgs }}$ is the subgrid Mach number. This is less than one for values of $\gamma$ encountered and ranging from 1.4 to 1.2. So it is not a bad approximation to replace the bar-filtered state equation by

$$
\varpi \approx \bar{\rho} R \vartheta
$$

which may hold even at high average Mach numbers. The resolved total energy is

$$
\bar{\rho} \tilde{e}=\bar{\rho} C_{v} \vartheta+\frac{1}{2} \bar{\rho}\left(\tilde{u}_{1}^{2}+\tilde{u}_{2}^{2}+\tilde{u}_{3}^{2}\right)
$$


Finally, the exact form of the bar-filtered fluxes can be written as

$$
\overline{\mathbf{F}_{\mathbf{i}}} \simeq\left(\begin{array}{l}
\bar{\rho} \tilde{u}_{i} \\
\bar{\rho} \tilde{u}_{i} \tilde{u}_{1}+\varpi \delta_{i 1}-\tau_{i 1}-2 \overline{\mu S_{i 1}} \\
\bar{\rho} \tilde{u}_{i} \tilde{u}_{2}+\varpi \delta_{i 2}-\tau_{i 2}-2 \overline{\mu S_{i 2}} \\
\bar{\rho} \tilde{u}_{i} \tilde{u}_{3}+\varpi \delta_{i 3}-\tau_{i 3}-2 \overline{\mu S_{i 3}} \\
(\bar{\rho} \tilde{e}+\varpi) \tilde{u}_{i}-Q_{i}-2 \overline{\mu u_{i} S_{i j}}-\overline{k \partial T / \partial x_{i}}
\end{array}\right)
$$

in which $Q_{i}$ is the $i$ th component of the subgrid heat-flux vector:

$$
Q_{i}=\overline{(\rho e+p) u_{i}}+(\bar{\rho} \tilde{e}+\varpi) \tilde{u}_{i}
$$

Bar-filtering equation (1) yields

$$
\frac{\partial \overline{\mathbf{U}}}{\partial t}+\frac{\partial \overline{\mathbf{F}_{\mathbf{i}}}}{\partial x_{i}}=0
$$

2.1.1 Subgrid-scale model. We will close the system described above by modelling the unknown terms as:

$$
\begin{aligned}
\tau_{i j}+2 \overline{\mu S_{i j}} & \simeq 2 \bar{\rho}\left(v_{t}+v\right) \tilde{S}_{i j} \\
Q_{i}+2 \overline{\mu u_{i} S_{i j}}+\overline{k \partial T / \partial x_{i}} & \simeq 2 \bar{\mu} \tilde{u}_{i} \tilde{S}_{i j}+\bar{k} \partial \vartheta / \partial x_{i}+\bar{\rho} C_{p} \frac{v_{t}}{\operatorname{Pr}_{\mathrm{t}}} \frac{\partial \vartheta}{\partial x_{i}}
\end{aligned}
$$

The subgrid-scale eddy coefficients are determined thanks to the filtered structure function (FSF) model which writes as

$$
v_{t}^{\mathrm{FSF}}(\vec{x}, t)=0.0014 C_{\mathrm{K}}^{-\frac{3}{2}} \Delta\left[\tilde{F_{2}}(\vec{x}, \Delta, t)\right]^{\frac{1}{2}}
$$

in which,

$$
\tilde{F_{2}}(\vec{x}, \Delta x, t)=\left\langle\left\|\mathcal{L}^{3}(\overline{\vec{u}}(\vec{x}+\vec{r}, t))-\mathcal{L}^{3}(\overline{\vec{u}}(\vec{x}, t))\right\|\right\rangle_{\|r\|=\Delta}
$$

is the second-order velocity structure function of the high-pass filtered velocity field $\mathcal{L}^{3}(\overline{\vec{u}})$. The filter $\mathcal{L}$ is a Laplacian operator approximated by second-order centred finite differences. $C_{\mathrm{K}}$ is the Kolmogorov constant and $\Delta$ is the mesh size. The turbulent Prandtl $P r_{\mathrm{t}}$ number is taken equal to 0.6 .

\section{Numerical solution and boundary conditions}

In this study we solve the compressible LES equations written in a Cartesian coordinate system, with $x$ (streamwise), $y$ and $z$ (transverse) directions. The numerical code employs a finite-difference MacCormack scheme [23], fourth-order in space and second-order in time [24]. The grid has $100 \times 74 \times 74$ points in a box of $35 \times 20 \times 20$ nozzle radii respectively in the longitudinal and orthogonal directions. In order to correctly simulate the jet shear layers, a non-uniform grid with a hyperbolic-tangent stretching is used in the $y$ and $z$ directions. This resolution of $\approx 550000$ points is moderate, and calculations have been carried out on a regular UNIX workstation. However, computing times necessary to have a converged solution with statistical stationarity are of the order of 40 hours, which is quite important. In fact, the objective of this work is to develop a LES code for a compressible jet which should be well validated against existing DNS and experiments, while remaining reasonable in terms of computing costs for users having only access to machines of this type. In this respect, and 
although convergence studies towards DNS by splitting the grid mesh size are desirable, they are not possible within this framework. Another appplication of this code will be to become a tool allowing control of the jet by proper deterministic upstream perturbations in order in particular to obtain bifurcating jets such as those simulated in [25] in the constant-density case. These control studies require many successive simulations since the upstream perturbations are changed. This cannot be done with a code much more expensive computationally if one keeps the strategy of using a workstation.

As far as the longitudinal dimension of the computational domain is concerned, let us stress that the ratio of the self-similar range length against the effective domain length is of about $77 \%$ in the subsonic case, and $50 \%$ in the supersonic case, as will be seen below. The ratio of the transverse jet shear-layer thickness against the transverse domain dimension is respectively $26 \%$ and $20 \%$ in both cases. Therefore, one can conclude that the computational domain size is sufficient. It does not seem to be too large, considering future control studies where the jet spreads out violently in the bifurcationg plane.

The special behaviour of compressible flows, such as acoustic wave reflection, requires an adequate treatment of boundary conditions. Otherwise, coherent vortices can be affected by spurious non-physical reflections and do not exit properly from the computational domain. For this purpose we have used a non-reflecting boundary condition proposed by Poinsot and Lele [26]. To absorb outgoing acoustic disturbances and turbulent structures, a sponge zone was set in the downstream part of the domain [27], of length $17 \%$ of the computational domain length. Boundary conditions based on the same principles have been used satisfactorily by many authors since then.

Morris [28] and Michalke and Hermann [29] found that the nature of the first instability is mainly conditioned by the mean velocity profile shape at the nozzle. Therefore, it is essential to simulate flow at the nozzle exit realistically. For the following simulations, the inflow longitudinal velocity is the same as for instability studies carried out in [29]:

$$
U_{\mathrm{m}}\left(\vec{x}_{0}\right)=\frac{U_{1}+U_{2}}{2}-\frac{U_{1}-U_{2}}{2} \tanh \left[\frac{1}{4} \frac{R}{\theta}\left(\frac{r}{R}-\frac{R}{r}\right)\right]
$$

where $U_{1}$ is the jet centreline, $U_{2}$ a small co-flow and $\theta$ the momentum thickness of the upstream shear layer. This profile does not simulate the flow inside the nozzle, but gives a good approximation of the inlet velocity profiles found in experimental measurements of round jets [30]. Notice that the inlet mean transverse velocity components are set to zero:

$$
V_{\mathrm{m}}\left(\vec{x}_{0}\right)=W_{m}\left(\vec{x}_{0}\right)=0
$$

In a compressible gas, any velocity gradient induces a heating due to molecular dissipation. Therefore, we associate a temperature profile (given by Crocco-Busemann's relation) to the upstream velocity profile.

Since the nozzle boundary layer has a dominant influence on the downstream jet development, the correct prescription of inflow perturbation is indeed very important. In the present study, we superpose a white noise upon the three velocity components in the shear-layer gradient. For all simulations carried out in this paper, the maximum amplitude of the random noise is set to $1.0 \%$ of $U_{1}$. One may assume that such a low intensity triggers jet natural development.

Compressibility is here characterized by the jet Mach number $M$, which is defined by means of the upstream jet-centre velocity $U_{1}$ and temperature $T_{1}$ :

$$
M=\frac{U_{1}}{\sqrt{\gamma R T_{1}}}
$$


The Reynolds number is based upon $U_{1}$ and the upstream jet diameter $D$ :

$$
R e=\frac{U_{1} \rho_{1} D}{\mu_{1}}
$$

$\mu_{1}$ and $\rho_{1}$ being the corresponding viscosity and density. We initiated all our simulations with the upstream profile, which is repeated at each $x$ up to the domain exit (temporal jet). Then the flow develops into a spatially growing jet up to a time $t_{\mathrm{s}}$ where it reaches statistical stationarity.

\section{Validation}

First we validate our code by comparison with a forced-jet DNS of Freund [31] and the experiment of Stromberg et al. [32] at $M=0.9$. The Reynolds number is moderate $(R e=$ 3600). The upstream velocity profile is the same as used by Freund:

$$
U\left(\overrightarrow{x_{0}}, t\right)=U_{\mathrm{m}}\left(\overrightarrow{x_{0}}\right)+U_{\text {for }}\left(\overrightarrow{x_{0}}, t\right)
$$

$U_{\mathrm{m}}\left(\overrightarrow{x_{0}}\right)$ being given by equation (28) in which $U_{1}=0.9, U_{2}=0$ and $R / \theta=11.2$. The upstream forcing $U_{\text {for }}\left(\overrightarrow{x_{0}}, t\right)$ is

$$
U_{\text {for }}\left(\overrightarrow{x_{0}}, t\right)=\epsilon U_{\mathrm{m}}\left(\overrightarrow{x_{0}}\right) \sin \left(S t_{D} \cdot t\right)
$$

in which $S t_{D}=f D / U_{1}=0.45$ is the forcing Strouhal number and $\epsilon=0.0025$ the amplitude. There is no forcing on the transverse velocity components.

Figure 1(a) shows the mean centreline velocity obtained from our LES at a time of $300 \mathrm{D} / U_{1}$ after $t_{\mathrm{s}}$. It is compared with data from [31] and [32]. Figure 1(b) shows the mean longitudinal velocity profiles for the same cases. There are some small differences between the data sets, but the general agreement is quite good, providing very good validation for our compressible LES code.
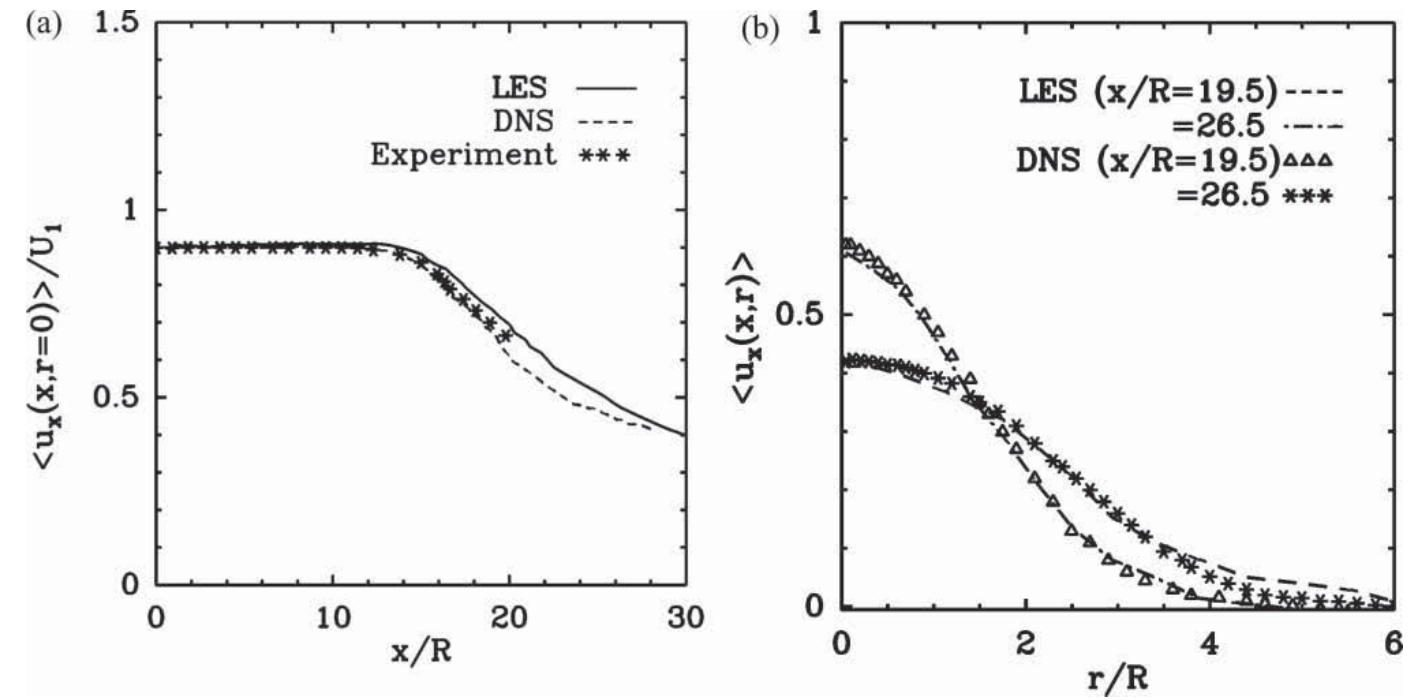

Figure 1. (a) Mean centreline velocity obtained at Mach 0.9 from the LES, DNS [31], and experiment [32]. (b) Mean velocity at two different downstream positions. 


\section{Compressibility effects in natural round jets}

Now we focus on the statistics and on the various coherent vortices present in free round jets at Mach 0.7 and 1.4.

For all the following simulations, the initial mean velocity is given by equation (28) with $R / \theta=20$, The initial centreline and co-flow jet velocities are $U_{1}=1.02$ and $U_{2}=0.02$. The co-flow is then very small $\left(U_{2} / U_{1}=0.0196<2 \%\right)$ and has only a minor influence on the jet dynamics.

\subsection{Subsonic jet $(\mathrm{M}=0.7)$}

As mentioned above, compressibility effects in compressible mixing layers are governed by the convective Mach number, which is $\approx 0.35$ here. This is well below the value of 0.6 at which compressibility effects begin to be important $[18,19]$. It is therefore acceptable to compare our simulation results with incompressible jet experiments carried out within a fully developed region. In this region, round jets obey the following relations [33]:

$$
\begin{aligned}
\frac{U_{1}-U_{2}}{\left\langle u_{x}(x, r=0)-U_{\text {cof }}\right\rangle} & =\frac{1}{B_{u}}\left[\frac{x-x_{0}}{D}\right] \\
\frac{\delta_{0.5}(x)}{D} & =C_{d}\left[\frac{x-x_{0}}{D}\right]
\end{aligned}
$$

where $\delta_{0.5}(x)$ is the jet shear-layer thickness, defined by

$$
\left\langle u_{x}\left(x, r=\delta_{0.5}(x)\right)\right\rangle-U_{\text {cof }}=0.5\left(\left\langle u_{x}(x, r=0)\right\rangle-U_{2}\right)
$$

$U_{\text {cof }}=\left\langle u_{x}(x, r=\infty)\right\rangle$ is the local co-flow, brackets \langle\rangle denote a time average and $x_{0}$ is the virtual origin of the jet (see figure 2 for definition). Figure 2 shows that the slope of the centreline velocity decay agrees well with the experimental data of [33]. To confirm this point, we have computed the centreline velocity decay rate $1 / B_{u}$ in the far-field region, and found $B_{u}=5.62$. This is very close to the value of $B_{u}=5.8$ obtained by Hussein et al.

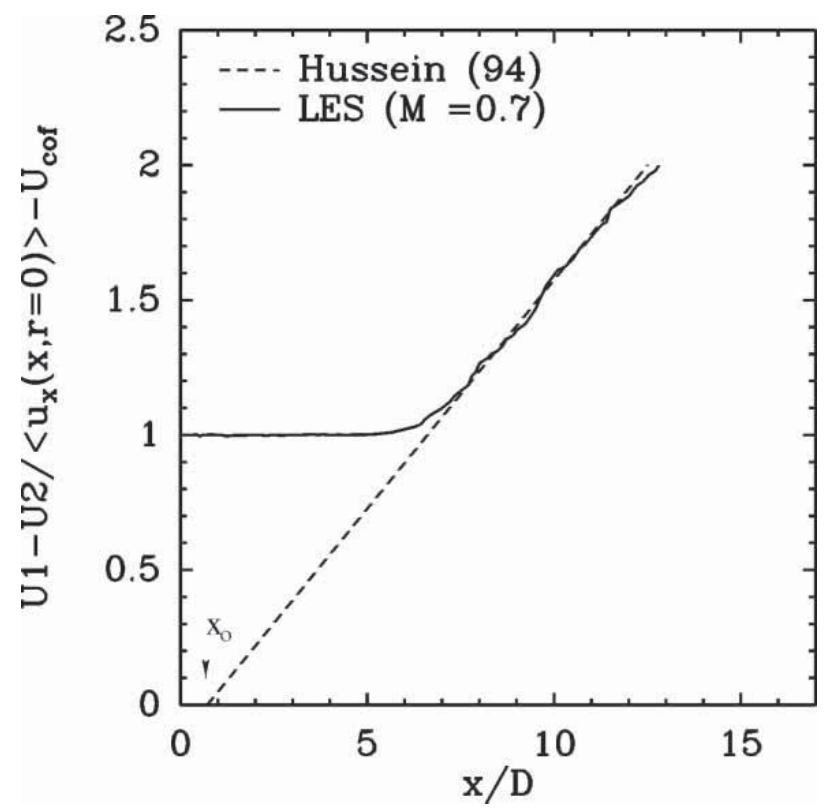

Figure 2. Streamwise evolution of the centreline velocity decay. 
(a)

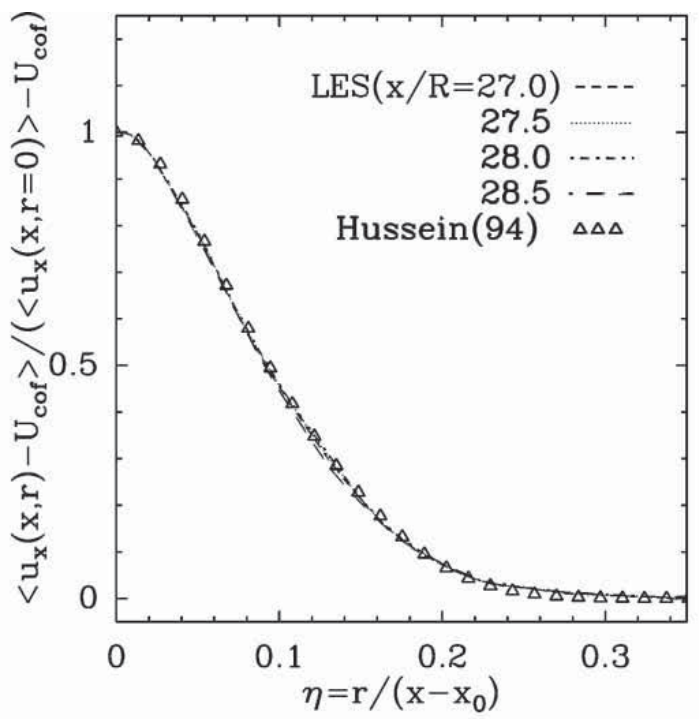

(b)

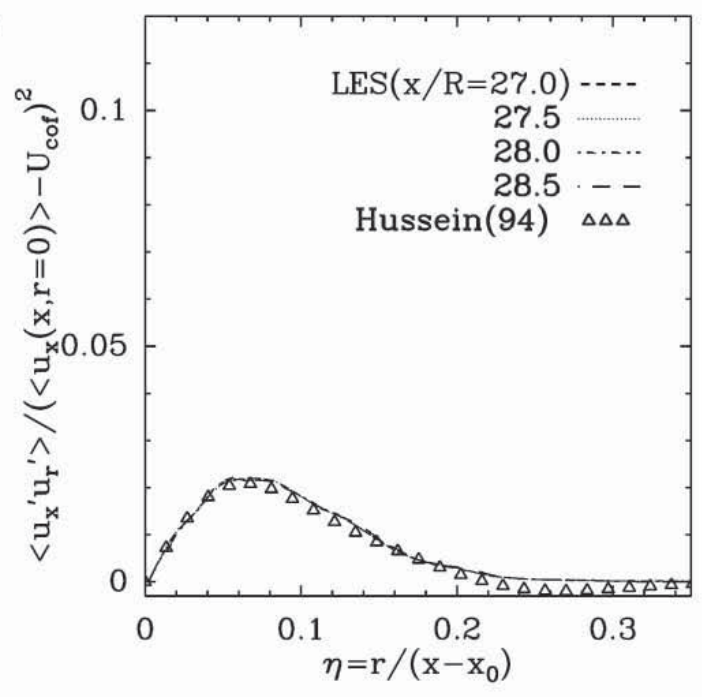

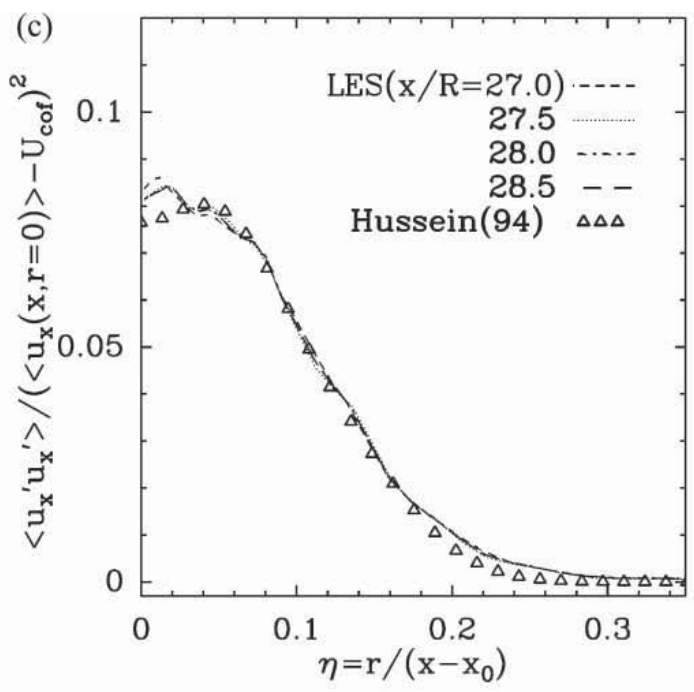

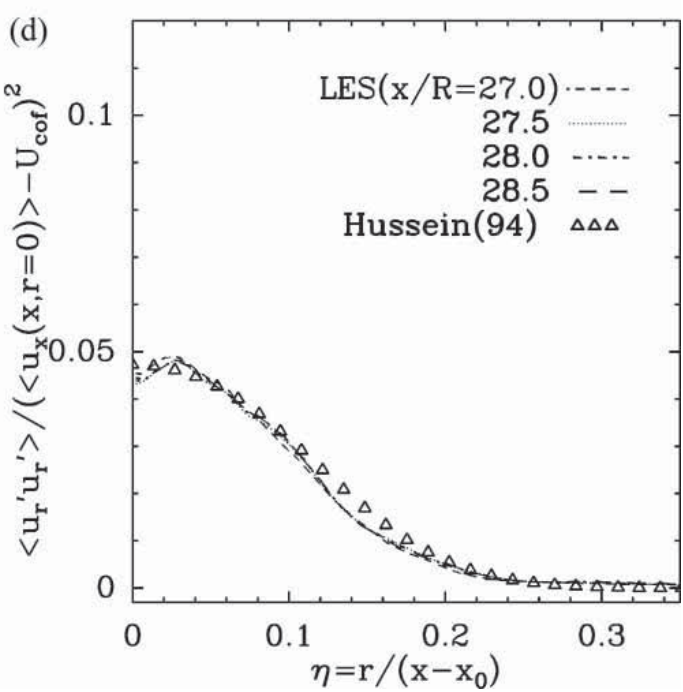

Figure 3. (a) Streamwise velocity profiles in the far field of the natural round jet at $M=0.7$ where $U_{\text {cof }}$ is the local co-flow velocity and $x_{0}$ is the virtual jet origin; (b) streamwise normal Reynolds stresses; (c) streamwise radial stresses; (d) radial stresses.

[33]. Figure 3(a) shows the centreline mean velocity profiles at several downstream locations obtained from our LES model, compared with incompressible experimental data [33]. The good superposition of the various self-similar experimental and numerical profiles validates our LES model. Notice, however, that laboratory experiments are not always carried out exactly as numerical simulations in terms of boundary conditions.

We have also determined the Reynolds stresses. Figures 3(b), 3(c) and 3(d) display the profiles of the streamwise normal, streamwise radial and radial components of Reynolds stress tensor, respectively. Overall, the agreement with experimental self-similar data is very good. Nevertheless the streamwise normal components (see figure 3(b)) are slightly overpredicted for a radial distance smaller than $\eta=r /\left(x-x_{0}\right)<0.03$. Streamwise radial cross stresses and radial stresses exhibit a very good agreement with the experimental data (figures 3(c) and $3(\mathrm{~d})$ ).

We now study the spatial evolution of coherent vortices present in the transitional region of the subsonic round jet. To visualize the jet structures, isosurfaces of positive $Q$ are used [34, 35], where $Q$ is the second invariant of the velocity gradient tensor, $Q=1 / 2\left(\Omega_{i j} \Omega_{i j}-\right.$ $S_{i j} S_{i j}$ ). We show here jets obtained for $R e=36000$. Figures 4(a) (at $t=30 \mathrm{D} /\left(U_{1}-U_{2}\right)$ ) 


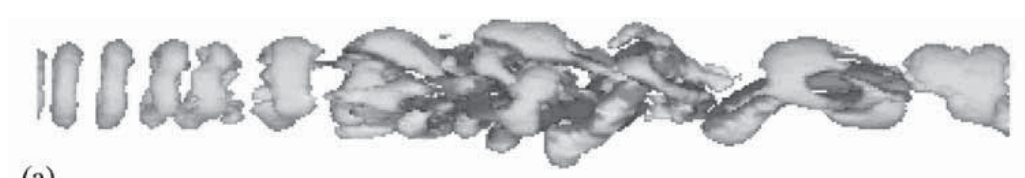

(a)

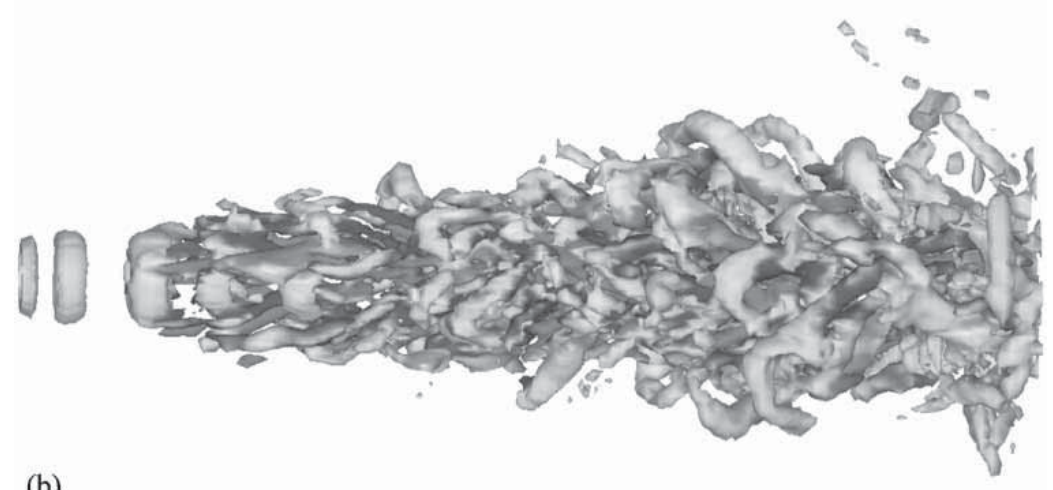

(b)

Figure 4. Isosurfaces of positive $Q$ (in green) and longitudinal vorticity (positive, red; negative, blue) for the subsonic jet at $M=0.7$. The threshold is $0.20\left(U_{1} / D\right)^{2}$. $R e=36000$. (a) $t=30 D /\left(U_{1}-U_{2}\right)$. (b) $t=300 D /\left(U_{1}-U_{2}\right)$. Animation 1 corresponds to case (b).

and (b) (at $t=300 \mathrm{D} /\left(U_{1}-U_{2}\right)$ ) present two different times of evolution of the flow, still at $M=0.7$. The flow field of figure 4(a) corresponds to a transient stage close to the initial computational time. For both cases the shedding of axisymmetric vortex rings is observed upstream. These axisymmetric coherent vortices represent the emergence of the so-called varicose mode. Concentrating on figure 4(a), one sees that the vortex rings move downstream while merging with neighbouring rings, so that the scale of the vortex rings increases with downstream distance. We have checked that the axisymmetric vortex rings persist up to $x / D \approx$ 6. Pairs of counter-rotating streamwise vortices (hairpin vortices) are stretched between them. These hairpin vortices have been observed experimentally at moderate Reynolds numbers by Lasheras and Meiburg [36] and Liepmann and Gharib [37]. Further downstream, $(x / D>6)$ they grow through an alternate pairing process. Such a pairing mode corresponds to the growth of a subharmonic perturbation developing after the formation of the primary vortex rings (see [25]). This vortex arrangement was also experimentally observed by Broze and Hussain [38].

Figure 4(b), complemented by animation 1, indicates the vortex-tori shedding upstream. Then, at a downstream distance of $\approx 2 D$, they stretch alternate hairpin-shaped streamwise vortices providing a $3 \mathrm{D}$ perturbation which triggers the alternate pairing. We will see below that the potential-core length is $\approx 5.5 D$, and corresponds to the point where the jet starts spreading self-similarly. Then the jet dynamics is a chaotic mixing of large-scale vortices undergoing various interactions (including helical pairing) and small-scale turbulence.

\subsection{Supersonic jet $(\mathrm{M}=1.4)$}

We now study the supersonic jet at Mach 1.4 and Reynolds 36000 . Figure 5 , taken at $t=300 t_{0}$ with $\left(t_{0}=D /\left(U_{1}-U_{2}\right)\right.$, corresponds to a statistically converged state. It is complemented by animation 2. Compared with figure 4(b), it shows that the vortex structures are very different from those existing at Mach 0.7. Indeed, the upstream axisymmetric mode has disappeared and the structures seem to undergo an alternate pairing immediately downstream of the nozzle. This is associated with a strong initial reduction of the jet spreading rate. This is in good agreement with previous experimental visualizations [17-19, 39]. Further downstream, the jet becomes suddenly more three-dimensional and, as will be confirmed in the next section, 


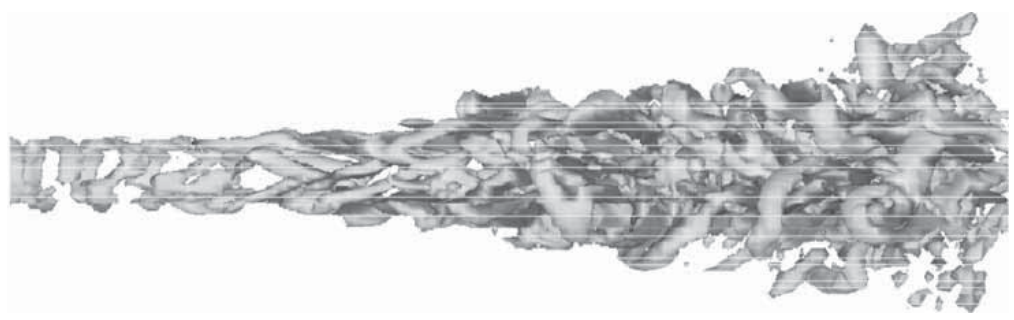

Figure 5. Isosurfaces of positive $Q$ (green) and longitudinal vorticity (red and blue) for the supersonic jet at $M=1.4$ and $R e=36000$. The threshold is $0.20\left(U_{1} / D\right)^{2}$. The figure corresponds to animation 2 .

spreads with a rate close to that of the subsonic jet. However, at a given downstream distance, the supersonic jet is shown to be more focused than the subsonic jet.

In the region close to the nozzle, the alternate pairing gives rise to strong alternate quasilongitudinal vortices. These observations are in agreement with visualizations of low-pressure isosurfaces carried out by Freund et al. [7]. They observed structures increasingly elongated in the flow direction when the Mach number increases; their convective Mach number goes from $M_{\mathrm{c}}=0.21$ to $M_{\mathrm{c}}=1.29$.

In fact, we recall that Fouillet's DNS [15] of natural compressible plane temporal mixing layers (presented in [40]) show an inhibition of helical pairing above a convective Mach number of 0.6-0.7. In the supersonic round jet at Mach 1.4, where the associated convective Mach number is equal to 0.69 , we have an important alternate pairing. If we identify the two types of pairing, which are both subharmonic, one would expect an inhibition of alternate pairing in the jet at higher Mach.

Figure 6 is a zoom of the jet in the transition region between $2 R$ and $5 R$, showing both $Q$ and longitudinal vorticity. It indicates the production (by vortex stretching of longitudinal vorticity) of both signs in the zones of vortex reconnection. This is also visible in figure 7(b) which shows in this region a typical plot of positive $Q$ isosurfaces coloured by longitudinal vorticity, seen from downstream. It is compared with the subsonic calculation (figure 7(a)). The latter displays very clearly the tore-shaped vortex without longitudinal vorticity.

\subsection{Compressibility effects upon statistics}

In order to reach good statistical convergence, the statistical study was carried out during one period of $300 \mathrm{D} /\left(U_{1}-U_{2}\right)$. This corresponds to twenty times the time required for a vortex to cross the physical domain.

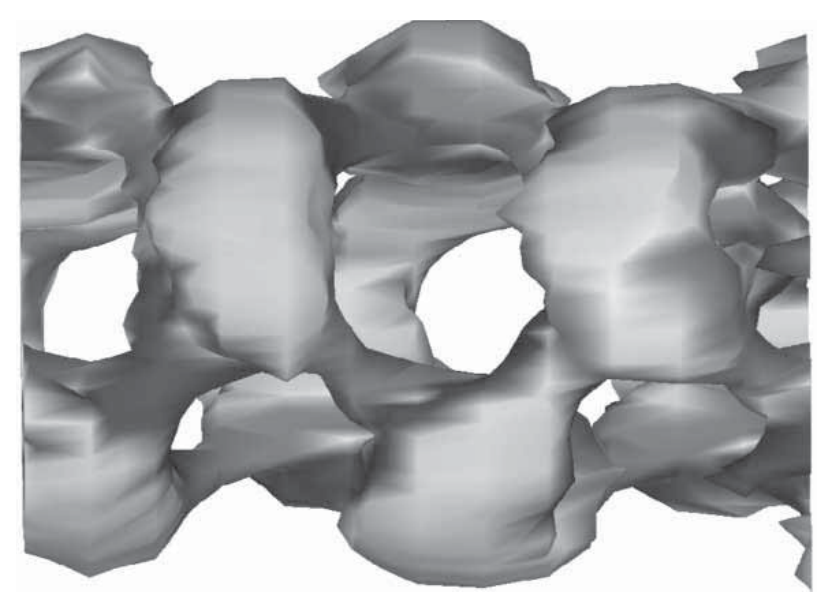

Figure 6. Isosurfaces of positive $Q$ (green) and longitudinal vorticity (red and blue) for the supersonic jet at $M=1.4$ and $2 \leq x / R \leq 5$. The threshold is $0.05\left(U_{1} / D\right)^{2}$. 

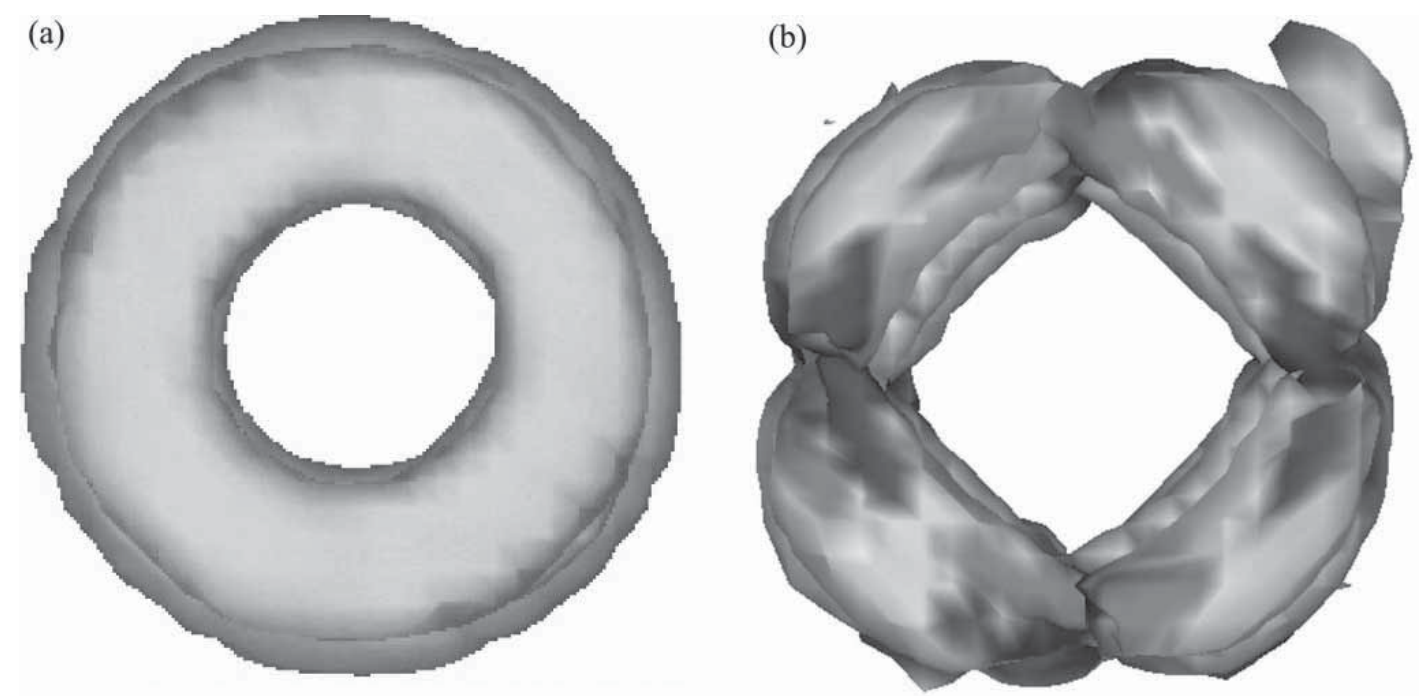

Figure 7. Isosurfaces of positive $Q$ (green) and longitudinal vorticity (red and blue) seen from downstream in the initial region $(1 \leq x / R \leq 5)$. The threshold is $0.15\left(U_{1} / D\right)^{2}$. (a) $M=0.7$. (b) $M=1.4$.

5.3.1 Mean flow statistics. Figure 8(a) shows the centreline streamwise velocity at Mach number 0.7 and 1.4. The fall around $x / D=5.5$ at Mach 0.7 indicates the end of the potentialcore. In this case, the potential-core length is therefore $5.5 D$. It becomes $7 D$ for a Mach number of 1.4 , representing an important increase of $27 \%$.

The spatial evolution of the jet shear-layer thickness is presented in figure 8(b). One can see that for both Mach numbers, the jets spread with the same rate up to $x / D \approx 5.5$. Beyond that, there is a sudden increase of the jet spreading rate, which is associated with an abrupt transition to three-dimensional developed turbulence. The supersonic jet spreads at a lower rate up to $x / D \approx 10$, then its spreading rate becomes comparable to the subsonic one. In fact, the local Mach number of the jet at a downstream distance $x$, defined by the jet centreline and the local sound speed, decreases with the jet centreline velocity. This is clear from figure 9 showing that the Mach 1.4 jet in fact becomes subsonic at a distance $x \approx 11 D$. This corresponds, neglecting possible corrections due to temperature variation, to the distance where its velocity is the upstream velocity divided by 1.4. Further downstream, the jet will be subsonic.
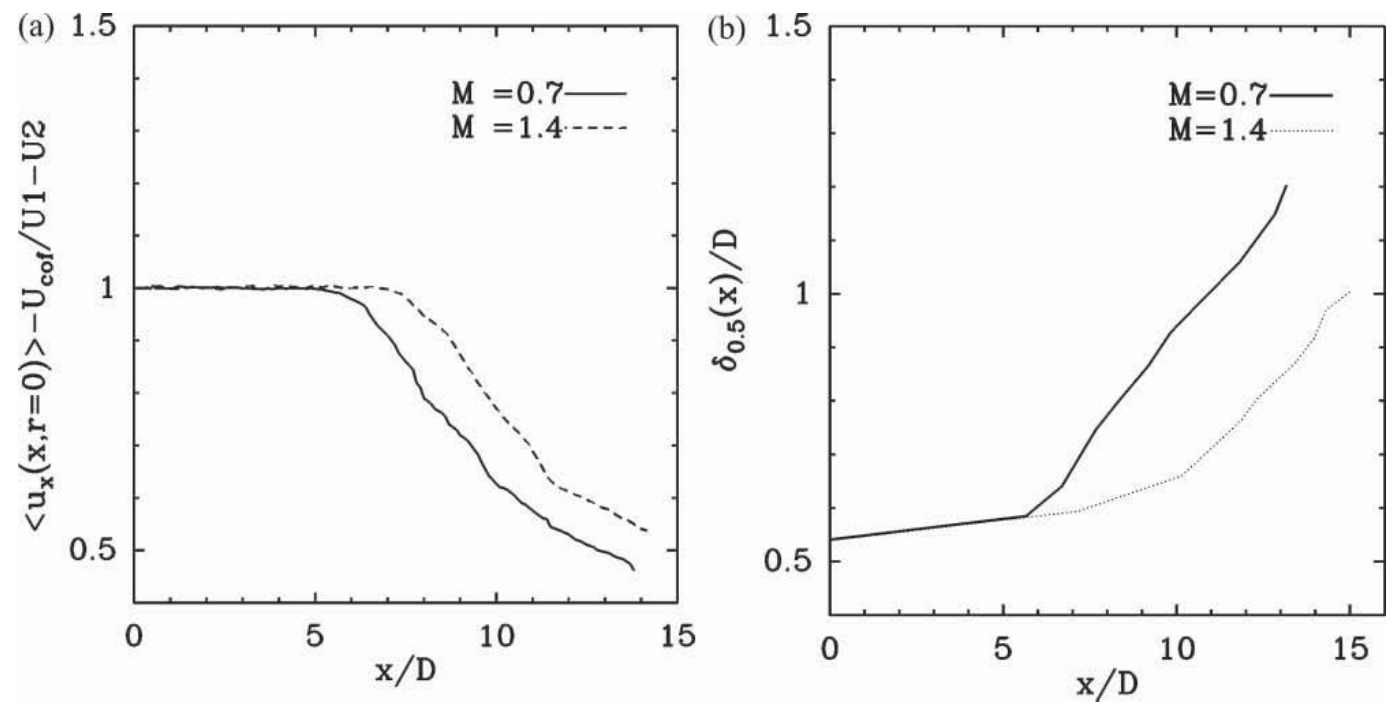

Figure 8. (a) Streamwise evolution of the mean centreline velocity for the natural jet at $M=0.7$ and $M=1.4$. (b) Streamwise evolution of the shear-layer thickness. $U_{\text {cof }}$ is the local co-flow velocity. 


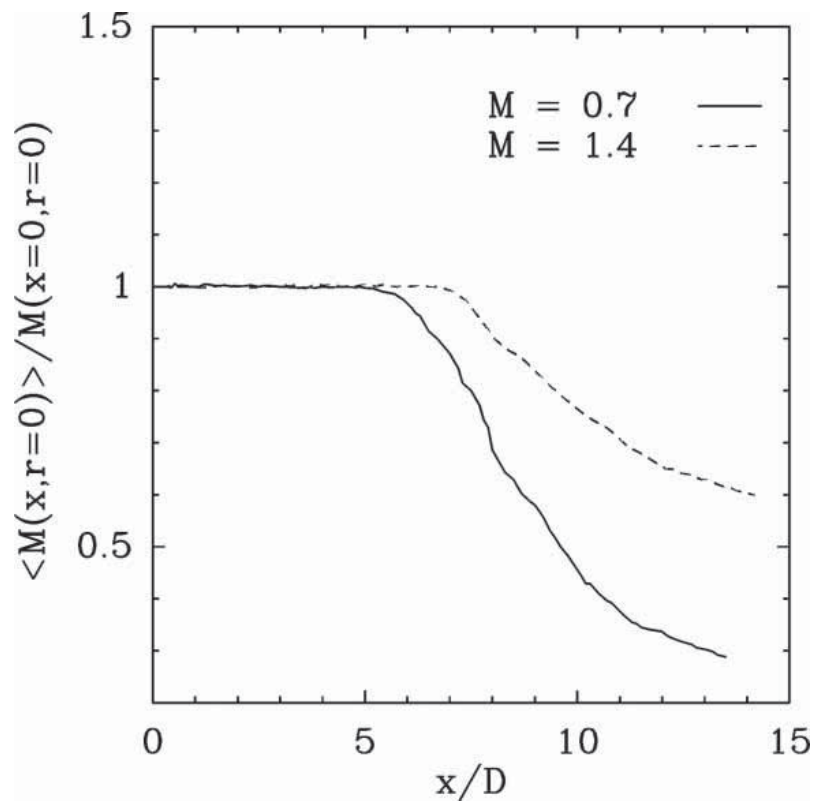

Figure 9. Streamwise evolution of the mean centreline Mach number for the natural jet at $M=0.7$ and $M=1.4$.

5.3.2 Reynolds stresses. In addition to the reduction in upstream jet spreading rate noticed at Mach 1.4, figure 10 indicates that compressibility also affects turbulent fluctuations and Reynolds stresses. In this figure, the Favre-averaged Reynolds stresses for the two Mach numbers are computed at the end of the jet potential core. It is clear that the streamwise radial $\langle\rho\rangle \widetilde{u_{x}^{\prime} u_{r}^{\prime}}$, radial $\langle\rho\rangle \widetilde{u_{r}^{\prime} u_{r}^{\prime}}$ and azimuthal $\langle\rho\rangle \widetilde{u_{\theta}^{\prime} u_{\theta}^{\prime}}$ stresses are reduced with increasing Mach number. On the contrary, the streamwise normal (axial) stress $\langle\rho\rangle \widetilde{u_{x}^{\prime} u_{x}^{\prime}}$ (when normalized by $\left.\rho_{1} U_{1}^{2}\right)$ is not reduced, and is very slightly increased. These results are qualitatively in good agreement with available experimental data [19, 41, 42] and the temporal numerical simulation of Freund et al. [7]. Notice however that, in figure 10, self-similarity is not yet achieved, contrary to the above-quoted laboratory experiments. Note also that the experiments of Samimy and Elliott [5, 6] showed a reduced axial normal stress as the Mach number increases.
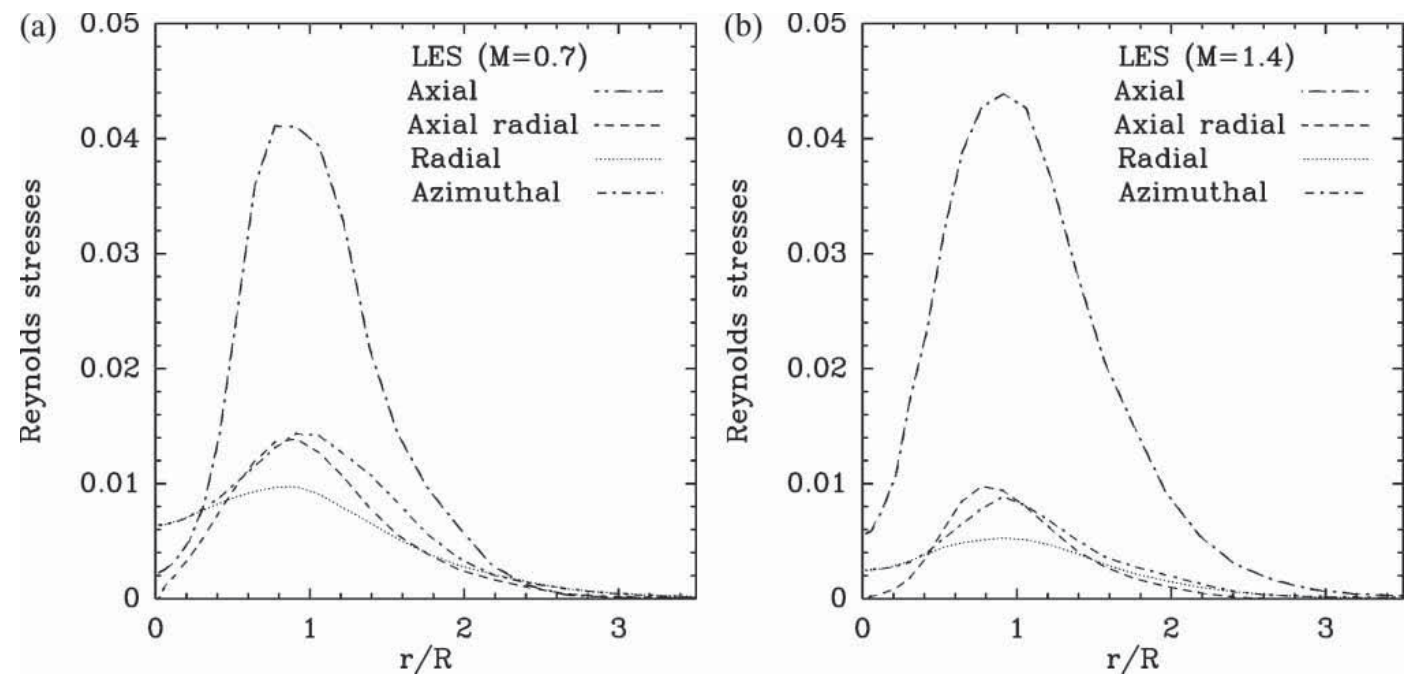

Figure 10. Favre-averaged Reynolds stresses (normalized by $\rho_{1} U_{1}^{2}$ ) profiles at the end of the potential core: (a) subsonic jet $(M=0.7)$; (b) supersonic jet $(M=1.4)$. 


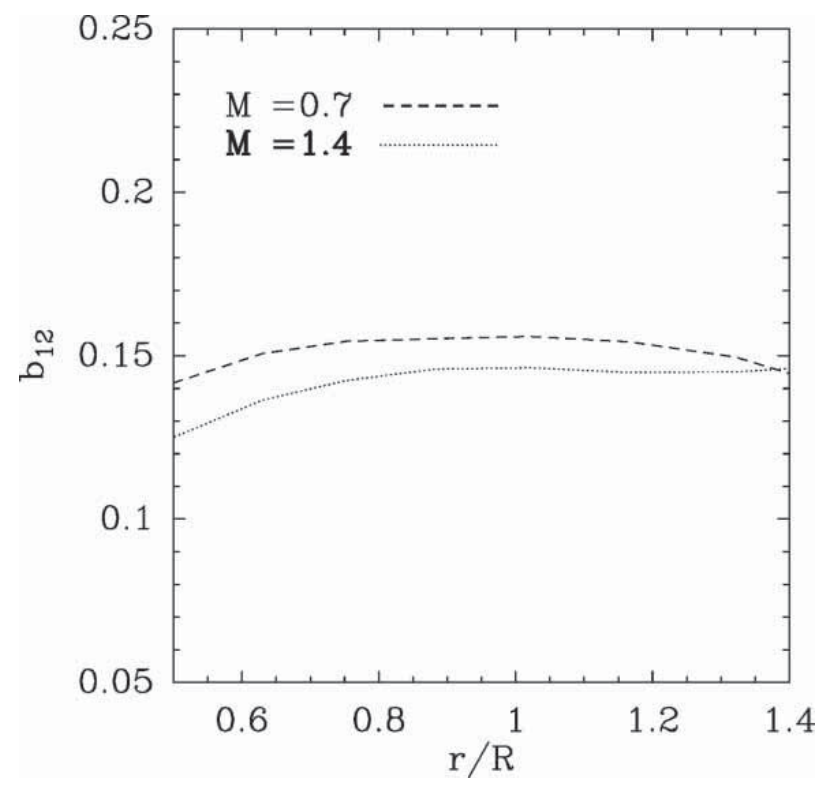

Figure 11. Shear-stress anisotropy profiles for the subsonic and supersonic jet.

5.3.3 Anisotropy tensor. The Reynolds stress reduction in the supersonic case can also be seen in figure 11 (still at the end of the jet potential core). This figure shows the shear-stress anisotropy parameter defined as follows:

$$
b_{12}=\frac{\widetilde{u_{x}^{\prime} u_{r}^{\prime}}}{2 K}
$$

where $K=1 / 2 \widetilde{u_{i}^{\prime} u_{i}^{\prime}}$ is the turbulent kinetic energy. Sarkar [20] proposed that the structural change in homogeneous shear flow turbulence at high compressibility is due to a change in magnitude of $b_{12}$, which implies a less efficient production mechanism. It turns out from this figure that the shear-stress anistropy across the middle of the mixing region decreases with increasing Mach number. This is in agreement with previous numerical results [7, 20]. Thus, the supersonic jet mixing layer has a lower production level than the subsonic one. According to Sarkar [20] (see also [43]), this reduction in turbulence production level is also responsible for the inhibition of turbulence growth rate. But these statistical results are in fact the consequence of the modification of the compressible mixing layer structure due to the inhibition of Kelvin-Helmholtz instability and predominance of three-dimensional quasilongitudinal instabilities.

To provide a detailed analysis of flow anisotropy, we have considered the anisotropyinvariant map proposed by Lumley and Newman [44]. This map uses the anisotropy tensor to characterize the turbulence state of the fluid flow. The anisotropy tensor is deduced from the Reynolds stress tensor by

$$
b_{i j}=\frac{\widetilde{u_{i}^{\prime} u_{j}^{\prime}}-2 / 3 k \delta_{i j}}{2 K}
$$

which enables us to have the three following invariants I, II, III:

$$
\begin{aligned}
I & =b_{i i} \\
I I & =-b_{i j} b_{j i} / 2 \\
I I I & =b_{i j} b_{j k} b_{k i} / 3
\end{aligned}
$$



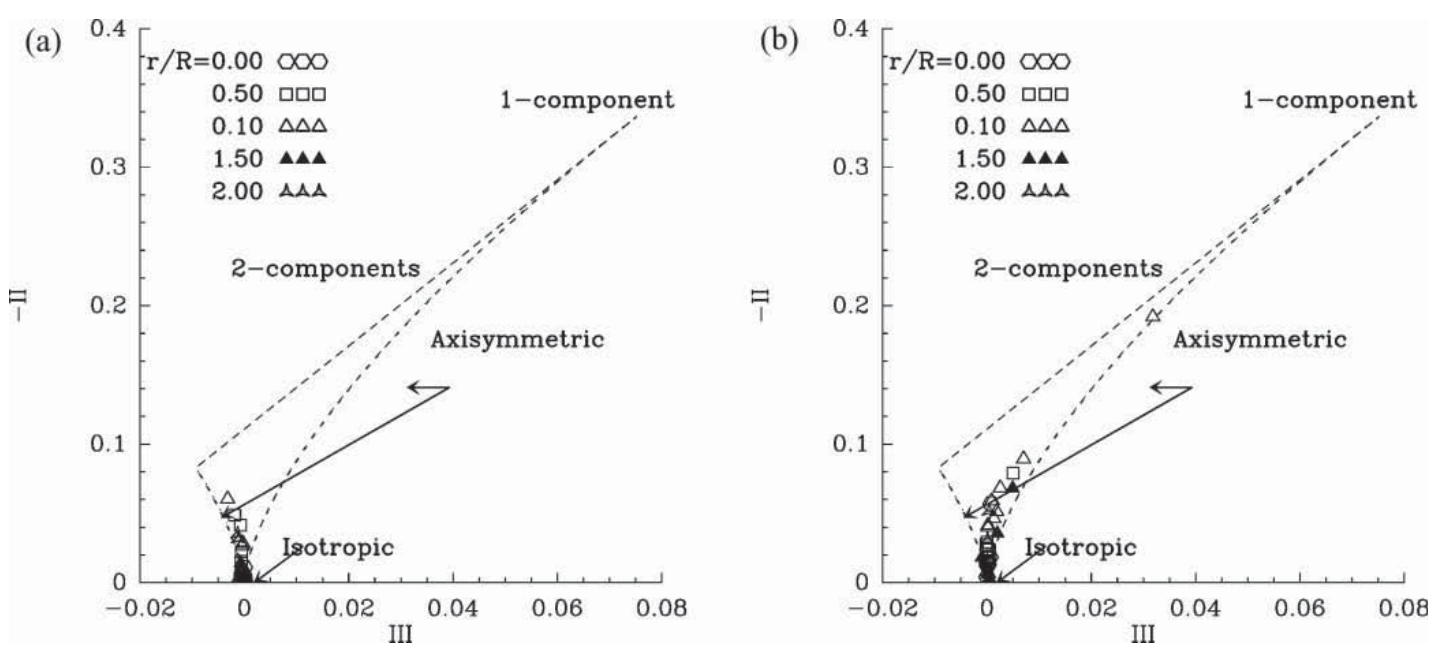

Figure 12. Anisotropy invariant map of the jet at: (a) $M=0.7$; (b) $M=1.4$. The invariants $I I$ and $I I I$ are computed at the centerline $(r / D=0)$, and for downstream locations range from $x / D=0$ to $x / D=14$.

These invariants are plotted on figures 12(a) and (b), which show that the flow returns to isotropy faster in the subsonic case than in the supersonic one. According to figure 12(a), the subsonic jet dynamics is dominated by two-dimensional large-scale structures in which the radial perturbation is preponderant. This two-dimensionality, associated with the shedding of vortex rings, disappears in the supersonic case, in which there is a dominance of streamwise perturbations.

5.3.4 Radial and azimuthal development of instabilities. In order to analyse the development of instabilities in the transitional zone of the jet, we have calculated the two following quantities

$$
\begin{aligned}
& E_{r}(x)=\sqrt{\frac{1}{L_{y} L_{z}}\left(2 \pi \int_{0}^{r_{\max }}\left\langle u_{r}^{\prime 2}(x, r)\right| r \mathrm{~d} r\right)} \\
& E_{\theta}(x)=\sqrt{\frac{1}{L_{y} L_{z}}\left(2 \pi \int_{0}^{r_{\max }}\left\langle u_{\theta}^{\prime 2}(x, r)\right| r \mathrm{~d} r\right)}
\end{aligned}
$$

which represent, respectively, the contribution of the radial and azimuthal Reynolds stresses to turbulent kinetic energy for a given position $x$. Here $r_{\max }=\sqrt{y_{\max }^{2}+z_{\max }^{2}}$ is the maximum radial distance from the jet axis.

Figures 13(a) and 13(b) represent the streamwise evolution of $E_{r}$ and $E_{\theta}$ for $M=0.7$ and $M=1.4$. It is clear that in the subsonic case, $E_{r}$, the quantity associated with the primary instabilities and the formation of vortex rings [45, 46] is largely dominant until the potentialcore end $(x / D \approx 5.5)$.

In agreement with the visual observations discussed above, this result demonstrates the dominance of ring-like structures in the transitional regime downstream of the nozzle. On the other hand, for the supersonic case (figure 13(b)), $E_{r}$ is dominated by $E_{\theta}$ until $(x / D \approx 7)$, with seems to be associated with the strong alternate pairing observed.

5.3.5 Radial lengthscale. The characteristic scales of the flow are very important as they are used for the statistical quantities scaling. Freund et al. [7] showed that turbulence statistics do not scale with the velocity difference across the mixing layer. Indeed, the normalization by 

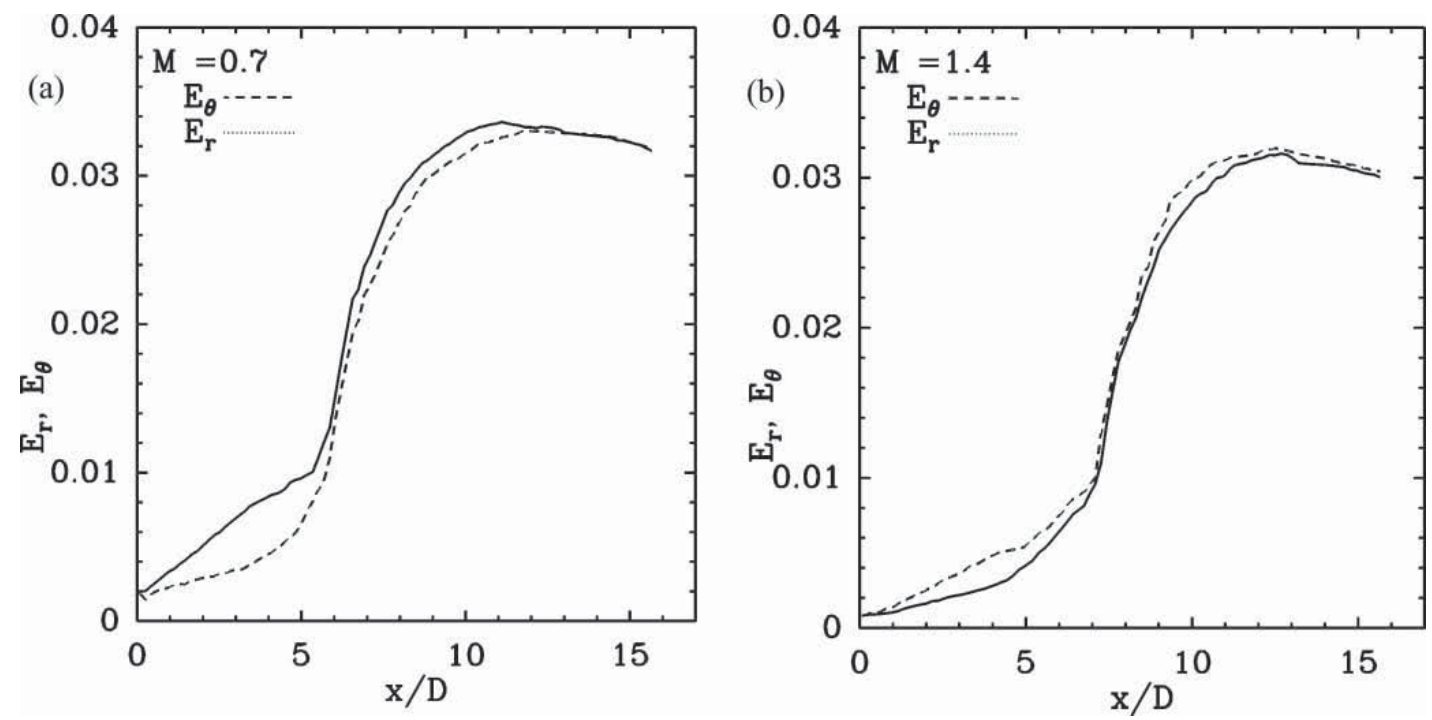

Figure 13. Longitudinal evolution of $E_{r}$ and $E_{\theta}$ at (a) $M=0.7$ and (b) $M=1.4$.

$\Delta U=U_{1}-U_{\text {cof }}(x)$, valid in the subsonic case, does not apply in the supersonic case in which the transverse (radial) lengthscale of large eddies, $l_{r}$, is less than the shear-layer thickness $\delta$ [7]. $U_{\text {cof }}(x)$ is the (local) streamwise velocity of the ambient fluid. In the compressible case, the statistics should scale with the velocity difference across a large eddy, $\Delta U l_{r} / \delta$.

Let us concentrate on the transverse large eddy lengthscale. It was defined by Freund et al. [47] in terms of the two-point correlation of radial velocity at $r=R$,

$$
\frac{\left\langle u_{r}^{\prime}\left(R-l_{r} / 2\right) u_{r}^{\prime}(R)\right\rangle}{\left\langle u_{r}^{\prime}(R) u_{r}^{\prime}(R)\right\rangle}=C_{l}
$$

and

$$
\frac{\left\langle u_{r}^{\prime}\left(R+l_{r} / 2\right) u_{r}^{\prime}(R)\right\rangle}{\left\langle u_{r}^{\prime}(R) u_{r}^{\prime}(R)\right\rangle}=C_{l}
$$

in which $C_{l}$ is a constant threshold . Freund et al. [47] proposed $C_{l}=0.5$. Here we choose to compute $l_{r}$ with a threshold value $C_{l}=0.45$. Changing the threshold of 0.45 to 0.5 has little effect on $l_{r}$ evaluation.

Figure 14 shows the downstream evolution of $l_{r}$ before the end of the potential core. It increases linearly with downstream distance. We have $\mathrm{d} l_{r} / \mathrm{d} x \approx 0.18$ in the supersonic case; 0.36 in the subsonic jet. This is in agreement with earlier DNS results [7] showing a decrease of transverse lengthscale with increasing Mach number in a turbulent round jet.

Previous experimental observations of free-shear flows such as round jets [17, 19, 39] and plane mixing layers $[9,10]$ indicate an inhibition of the interaction between turbulence structures (such as pairing phenomenon) with increasing compressibility. Indeed, the vortex pairing that occurs in the jet transitional region gives rise to largerscale structures and hence jet growth. These observations are supported by the present study, where pairing has been replaced by alternate pairing.

5.3.6 Fluid entrainment. As mentioned above, the large-scale structures of the jet set the irrotational ambient fluid in motion via the the Reynolds stresses. Therefore the stress reduction that occurs at high Mach numbers reduces this entrainment phenomenon. 


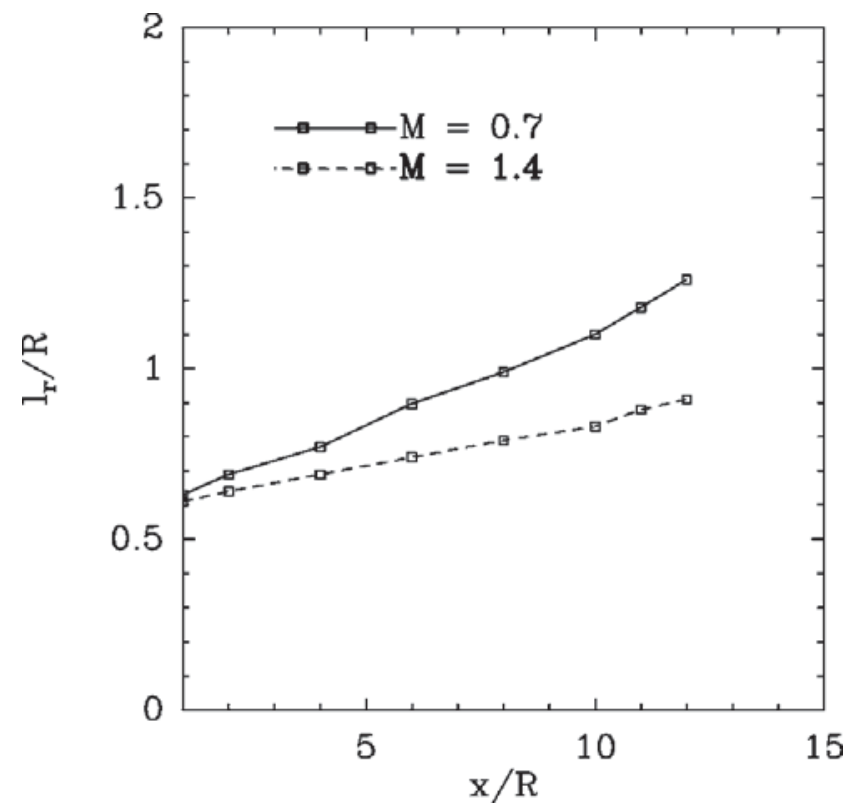

Figure 14. Radial large eddy lengthscale in the transitional zone of the subsonic and supersonic round jets.

Figure 15 compares the entrainment measurements of the subsonic jet with those of the supersonic jet in the transitional region. Jet entrainment measurements are based on evaluating the streamwise mass flux $Q(x)$, which is normalized with its upstream value at the nozzle $Q_{0}$ $[1,48,49]$. The mass flux $Q(x)$ is defined by a surface integral over the streamwise mean velocity and density. In agreement with previous observations and results, this figure confirms the decrease of jet entrainment with increasing Mach number. Indeed, the entrainment rate of the subsonic jet is significantly higher than that of the supersonic jet. In the subsonic case the entrainment rate is $\mathrm{d} Q / \mathrm{d} x=0.1 Q_{0} / D$ for $x \leq 6 D$ and $\mathrm{d} Q / \mathrm{d} x=0.265 Q_{0} / D$ for $x \geq 6 D$. Crow and Champagne [1] give, for incompressible jets, the following values: $\mathrm{d} Q / \mathrm{d} x=0.136 Q_{0} / D$ for $x \leq 2 D$ and $0.292 Q_{0} / D$ for $x \geq 6 D$, which are close. In our supersonic jet, the entrainment rate becomes $0.048 Q_{0} / D$ for $x \leq 6 D$, which corresponds to a decrease of $52 \%$ with respect to the subsonic jet. Let us mention the experimental work of Zaman [50] concerning jets in a range of Mach numbers between 0.3 and 2. His measurements

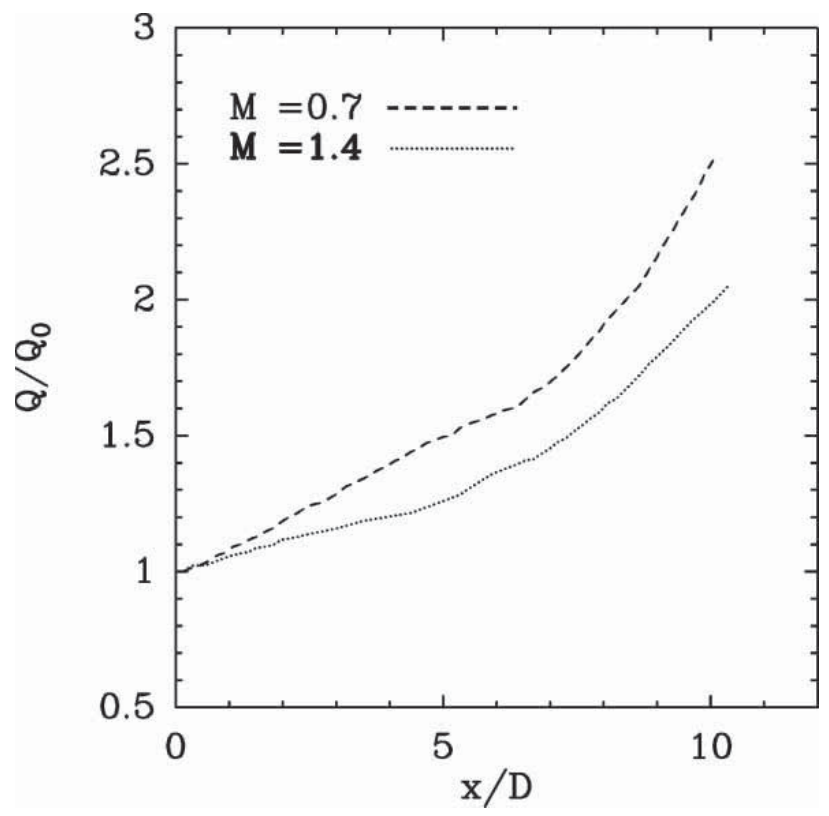

Figure 15. Downstream evolution of mass flux in the transitional zone of the subsonic and supersonic round jets. 
concern downstream distances larger than $10 D$. He also found a reduction of the entrainment rate with compressibility. LES in longer domains are required in order to make quantitative comparisons.

\section{Conclusions}

We have been able to simulate subsonic and supersonic turbulent round jets with the aid of large eddy simulations carried out on an orthogonal grid (stretched in the transverse directions) with MacCormack's finite-difference methods of fourth-order in space and second-order in time. The numerical resolution is moderate (550,000 grid points). We use the filtered structure function model. The jet is initiated upstream by a basic velocity profile close to a top hat, plus a weak co-flow and various small perturbations. The corresponding temperature profile is determined thanks to Crocco-Busemann's relation, and the jet Mach number is based upon the upstream centreline velocity and temperature.

We first validated our LES code by comparison with a forced-jet DNS at Reynolds number 3600 and Mach number 0.9 carried out by Freund [31], and an experiment by Stromberg et al. [32]. Agreement is very good, as far as the mean centreline velocity and the transverse mean velocity profiles are concerned.

After this validation, we considered jets at a higher Reynolds number $(36,000)$, forced by a weak random isotropic perturbation. Two Mach numbers were studied: in the subsonic $(M=0.7)$ and supersonic $(M=1.4)$ regimes.

The Mach 0.7 jet has a convective Mach number of 0.35 , and is weakly affected by compressibility. Close to the numerical nozzle, the jet sheds quasi-axisymmetric vortex rings with longitudinal hairpin vortices stretching between them, before the development of alternate pairing interactions which yield important jet widening and development to turbulence. Alternate pairing is, for the jet, the equivalent of helical pairing for a plane mixing layer. The centreline velocity, the transverse mean velocity and various Reynolds stress profiles compare very well in the far field with incompressible experiments of Hussein et al. [33].

The vortex rings shed from the nozzle at Mach 0.7 disappear at Mach 1.4. Instead, we have shown that the primary vortices undergoing alternate pairing form immediately. This phenomenon is associated with a dramatic initial reduction of the jet spreading rate, yielding a $27 \%$ increase of the jet potential-core length with respect to the subsonic case. Further downstream, the jet becomes subsonic and spreads out, as in the Mach 0.7 case. Comparison

of Lumley's anisotropy-invariant map at the centreline and for downstream location range from $x / D=0$ to $x / D=14$ has provided interesting informations on the jet structure, since, from this viewpoint, the Mach 0.7 jet is dominated by vortex rings while at Mach 1.4 there are mainly streamwise perturbations. Finally, the analysis of Reynolds stresses has indicated that turbulence intensities decrease with increasing Mach number, but with only a very weak effect on the streamwise normal stress. This is in good agreemennt with a decrease of the large-structure's radial lengthscale observed.

These results have important applications in active control of compressible jets dynamics, which will be studied in a forthcoming paper.

\section{Acknowledgement}

This work was supported by the Supersonic Research Programme of the French Ministry of Research (Technology Direction). 


\section{References}

[1] Crow, S.C. and Champagne, F.H., 1971, Orderly structure in jet turbulence. Journal of Fluid Mechanics, 48, 547-591.

[2] Zaman, K.B.M.Q. and Hussain, K.M.F., 1980, Vortex pairing in a circular jet under controlled excitation. Part 1. General jet response. Journal of Fluid Mechanics, 101, 449-491.

[3] Bogdanoff, D.W., 1983, Compressibility effects in turbulent shear layers. AIAA Journal, 21, 926-927.

[4] Papamoschou, D. and Roshko, A., 1988, The compressible turbulent shear layer: an experimental study. Journal of Fluid Mechanics, 197, 453-477.

[5] Elliott, G.S. and Samimy, M., 1990, Compressibility effects in free shear layers. Physics of Fluids A, 2, 1231-1130.

[6] Samimy, M. and Elliott, G.S., 1990, Effects of compressibility on the characteristics of free shear layers. AIAA Journal 28, 439-445.

[7] Freund, J.B., Lele, S.K. and Moin, P., 2000, Compressibility effects in a turbulent annular mixing layer. Pat 1. Turbulence and growth rate. Journal of Fluid Mechanics, 421, 229-267.

[8] Brown, G.L. and Roshko, A., 1974, On density effects and large structure in turbulent mixing layers. Journal of Fluid Mechanics, 64, 775-816.

[9] Papamoschou, D. and Bunyajitradulya, A., 1997, Evolution of large eddies in compressible shear layers. Physics of Fluids, 9, 756.

[10] Elliott, G.S., Samimy, M. and Arnette, S.A., 1995, The characteristics and evolution of large-scale structures in compressible mixing layers. Physics of Fluids, 7, 864-876.

[11] Beer, A., 2003, Etude par simulation numérique temporelle des effets de compressibilité en couche de mélange plane turbulente, $\mathrm{PhD}$ thesis, Grenoble: Institute National Polytechnique de Grenoble.

[12] Winant, C.D. and Browand, F.K., 1974, Vortex pairing: the mechanism of turbulent mixing-layer growth at moderate Reynolds number. Journal of Fluid Mechanics, 63, 237-255.

[13] Sandham, N.D. and Reynolds, W.C., 1989, The compressible mixing layer: linear theory and direct simulation. AIAA Paper, 89-0371.

[14] Sandham, N.D. and Reynolds, W.C., 1991, Three-dimensional simulations of large eddies in the compressible mixing layer. Journal of Fluid Mechanics, 224, 133-158.

[15] Fouillet, Y., 1992, Contribution à l'étude par expérimentation numérique des écoulements cisaillés libres, $P h D$ thesis, Grenoble: Institute National Polytechnique de Grenoble.

[16] Comte, P., Lesieur, M. and Lamballais, E., 1992, Large- and small-scale stirring of vorticity and a passive scalar in a 3-D temporal mixing layers, Physics of Fluids A, 4, 2761-2778.

[17] Fourguette, D.C., Mungal, M.G. and Dibble, R.W., 1991, Time evolution of the shear layer of a supersonique axisymmetric jet at matched conditions. AIAA Journal, 29, 1123-1130.

[18] Clemens, N.T. and Mungal, M.G., 1995, Large-scale structure and entrainment in the supersonic mixing layer. Journal of Fluid Mechanics, 284, 171-216.

[19] Thurow, B., Samimy, M. and Lempert, W., 2003, Compressibility effects on turbulence structures of axisymmetric mixing layers. Physics of Fluids, 15, 1755-1765.

[20] Sarkar, S., 1995, The stabilizing effect of compressibility in turbulent shear flow. Journal of Fluid Mechanics, 282, 163-186.

[21] Simone, A., Coleman, G.N. and Cambon, C., 1997, The effect of compressibility on turbulent shear flow: a rapid-distortion-theory and direct-numerical-simulation. Journal of Fluid Mechanics, 330, 307-338.

[22] Lesieur, M. and Comte, P., 1997, Turbulence in compressible flow. AGARD/Von Karman Institute Course, AGARD Report 819.

[23] MacCormack, R.W., 1969, The effects of viscosity in hypervelocity impact cratering. AIAA Paper 69-354.

[24] Gottlieb, D. and Turkel, E., 1976, Dissipative two-four methods for time-dependent problems. Journal of Computational Physics, 30, 703-723.

[25] da Silva, C.B. and Métais, O., 2002, Vortex control of bifurcating jets: a numerical study. Physics of Fluids, 14, 3798-3819.

[26] Poinsot, T.J. and Lele, S.K., 1992. Boundary conditions for direct simulations of compressible viscous flows. Journal of Computational Physics, 101, 104-129.

[27] Sandhu, H.S. and Sandham, N.D., 1994, Boundary conditions for spatially growing compressible shear layers. Technical report, Department of Aeronautical Engineering, Queen Mary \& Westfield College.

[28] Morris, P.J., 1976, The spatial viscous instability of axisymmetric jets. Journal of Fluid Mechanics, 77, 511529.

[29] Michalke, A. and Hermann, G., 1982, On the inviscid instability of a circular jet with external flow. Journal of Fluid Mechanics, 114, 343-359.

[30] Freymuth, P., 1966, On transition in a separated laminar boundary layer. Journal of Fluid Mechanics, 25, 683-704.

[31] Freund, J.B., Lele, S.K. and Moin, P., 1999, Acoustic sources in a turbulent jet: a direct numerical simulation study. AIAA paper 99-1858.

[32] Stromberg, J.L., Mclaughlin, D.K. and Troutt, T.R., 1980, Flow field and acoustic properties of a Mach number 0.9 jet at low Reynolds number. Journal of Sound and Vibration, 72, 159-176.

[33] Hussein, H.J., Capp, S.P. and George, W.K., 1994. Velocity measurements in a high-Reynolds-number momentum-conserving, axisymmetric, turbulent jet. Journal of Fluid Mechanics, 258, 31-75. 
[34] Hunt, J.C.R., Wray, A.A. and Moin, P., 1988, Eddies, stream, and convergence zones in turbulent flows. Annual Research Briefs, Center for Turbulence Research, Stanford University, Stanford, CA.

[35] Dubief, Y. and Delcayre, F., 2000, On coherent-vortex identification in turbulence. Journal of Turbulence, 1(011), 1-22.

[36] Lasheras, J.C. and Meiburg, E., 1991, On the three-dimensional dynamics of the coherent vertical structures forming in free shear flows. In: O. Métais and M. Lesieur (Eds.) Turbulence and Coherent Structures. (Dordrecht: Kluwer).

[37] Liepmann, D. and Gharib, M., 1992, The role of the streamwise vorticity in the near-field entrainement of round jets. Journal of Fluid Mechanics, 245, 643-688.

[38] Broze, G. and Hussain, F., 1996, Transition to chaos in a forced jet: intermitency, tangent bifurcations and hysteresis. Journal of Fluid Mechanics, 311, 37-71.

[39] Naughton, J.W., Cattafesta, L.N. and Settles, G.S., 1997, An experimental study of compressible turbulent mixing enhancement in swirling jets. Journal of Fluid Mechanics, 330, 271-305.

[40] Lesieur, M., 1997, Turbulence in Fluids. (Dordrecht: Kluwer).

[41] Göebel, S.G. and Dutton, J.C., 1991, Experimental study of compressible turbulent mixing layers. AIAA Journal, 29, 538-546.

[42] Urban, W.D. and Mungal, M.G., 1998, Planar velocity measurements in compressible mixing layers. Journal of Fluid Mechanics, 431, 189-222.

[43] Pantano, C. and Sarkar, S., 2002, A study of compressibility effects in the high-speed turbulent shear layer using direct simulation. Journal of Fluid Mechanics, 451, 329-371.

[44] Lumley, J.L. and Newman, G.W., 1977, The return to isotropy of homogeneous turbulence. Journal of Fluid Mechanics, 82, 161-178.

[45] Brancher, P., Chomaz, J.M. and Huerre, P., 1994, Direct numerical simulations of round jets: vortex induction and side jets. Physics of Fluids, 6, 1768-1774.

[46] Danaila, I., Dusek, J. and Anselmet, F., 1997, Coherent structures in a round, spatially evolving, unforced, homogeneous jet at low Reynolds numbers. Physics of Fluids, 9, 3323-3342.

[47] Freund, J.B., Lele, S.K. and Moin, P., 1998, Direct simulation of a Mach 1.92 jet and its sound field. Paper presented at the 4th AIAA/CEAS Aeroacoustics Conference, June 2-4, Toulouse, France.

[48] Grinstein, F.F., 2001, Vortex dynamics and entrainment in rectangular free jets. Journal of Fluid Mechanics, 437, 69-101.

[49] Ho, C.M. and Gutmark, E., 1987, Vortex induction and mass entrainment in a small-aspect-ratio elliptic jet. Journal of Fluid Mechanics, 179, 383-405.

[50] Zaman, K.B.M.Q., 1999, Spreading characteristics of compressible jets from nozzles of various geometries. Journal of Fluid Mechanics, 383, 197-228. 